\title{
Controlling Energy Gap of Bilayer Graphene by Strain
}

\author{
Seon-Myeong Choi, ${ }^{1}$ Seung-Hoon Jhi, ${ }^{1,2}$, 田 and Young-Woo Son ${ }^{3}$, \\ ${ }^{1}$ Department of Physics, Pohang University of Science and Technology, Pohang 790-784, Korea \\ ${ }^{2}$ Division of Advanced Materials, Pohang University of Science and Technology, Pohang 790-784, Korea \\ ${ }^{3}$ Korea Institute for Advanced Study, Seoul 130-722, Korea
}

(Dated: July 13, 2018)

\begin{abstract}
Using the first principles calculations, we show that mechanically tunable electronic energy gap is realizable in bilayer graphene if different homogeneous strains are applied to the two layers. It is shown that the size of energy gap can be simply controlled by adjusting the strength and direction of these strains. We also show that the effect originates from the occurrence of strain-induced pseudoscalar potentials in graphene. When homogeneous strains with different strengths are applied to each layer of bilayer graphene, transverse electric fields across the two layers can be generated without any external electronic sources, thereby opening an energy gap. The results demonstrate a simple mechanical method of realizing pseudo-electromagnetism in graphene and suggest a maneuverable approach to fabrication of electromechanical devices based on bilayer graphene.

PACS numbers: 73.22.Pr, 77.65.-j, 62.25.-g, 81.05.ue
\end{abstract}

Materials that can change energy gaps under mechanical deformations are greatly desired to develop electromechanical nanodevices with controllable optical and electrical operational ranges [1,2]. Graphene, one atom thick semi-metallic membrane [3 5], may have this property because of its special electromechanical characteristics such as strain-induced phases in the chiral wavefunctions of the massless Dirac Hamiltonian [5-10]. Although external electric fields have been used to tune the energy gaps in several forms of graphene 11 20, a simple and practical method of tuning the electronic energy gaps based on mechanical operations is lacking.

The influence of smooth elastic deformations on the physics of a single layer of graphene can be described by introducing a suitable gauge-field vector potential to the free massless particle Dirac's equation [5]. The choice of vector potential depends on the direction and strength of applied strains. Specifically designed inhomogeneous strains can produce a net pseudo-magnetic flux that be expected to induce interesting electromagnetic effects $\underline{6}$ 10]. In contrast, under homogeneous uniaxial or biaxial strains, the effect of pseudo-magnetic fields is absent but a homogeneous pseudo-scalar potential can be generated owing to electron density variations or dilations [10, 2123]. Because graphene is a one atom thick semimetal, the pseudo-scalar potential will alter its work function significantly. When a uniaxial (or isotropic) strain is applied up to an experimentally realizable magnitude of $\sim 10 \%$, the work function of graphene is predicted to increase by 0.27 (or 0.65 ) $\mathrm{eV}$ from the value of $4.49 \mathrm{eV}$ of graphene in equilibrium [23]. Hence, the homogeneous strain can have important applications in various electronic devices for tuning the band lineup between metallic leads and graphene, or in changing the amount of charge transfer from foreign molecules. We also expect that such strain-induced pseudo-scalar potentials will modify the electronic structure of bilayer graphene significantly.
In this paper, we predict the formation of mechanically tunable electronic energy gap in bilayer graphene. It is shown that the perpendicular electric fields across the two layers of bilayer graphene can be generated without any external gate potential if each layer is subjected to different strengths of homogeneous strains. This effect originates from asymmetric generations of pseudoscalar potential in each layer of strained bilayer graphene, which alter their work functions significantly [13]. We also show that the strain-induced electronic energy gaps are indirect in general and strongly depend on the size and direction of applied homogeneous strains. We discuss origins of such peculiar variations of the bandgaps and present realistic experimental setups for observing the energy gaps.

Our study of electronic structures of strained bilayer graphene is based on the pseudopotential density functional method 24]. The exchange-correlation interactions were treated within the local density approximation [25]. The cutoff energy for expansion of wavefunctions and potentials was $400 \mathrm{eV}$. Extensive momentum space sampling in the whole Brillouin zone (BZ) is performed to obtain accurate energy dispersions, reliable energy gaps, and their dependences on strain. The Monkhorst-Pack $k$-point grid of $2 \times 12 \times 1$ is used for the atomic relaxation and of $5 \times 30 \times 1$ for electronic structure calculation of $11.1 \%$ asymmetrically strain bilayer graphene. Calculations for other strengths of strain also used an equivalent number of $k$-points. The atomic relaxation was carried out until the Helmann-Feynman forces were less than $0.03 \mathrm{eV} / \AA$. The distance between adjacent bilayer graphene in the supercells is $26 \AA$ avoiding spurious interactions between them. We also have increased the distance up to $36 \AA$ finding no changes in electronic structures. The weak dispersion force between the two layers of bilayer graphene is not considered here and the interlayer distance is fixed to $3.35 \AA$ because its 


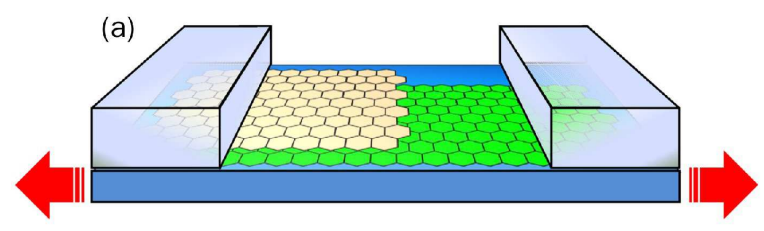

(b)

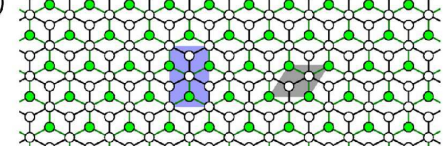

(c)

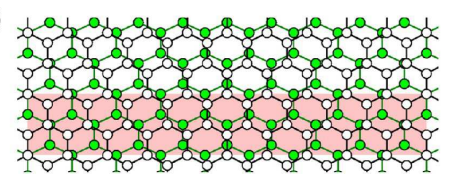

(d)
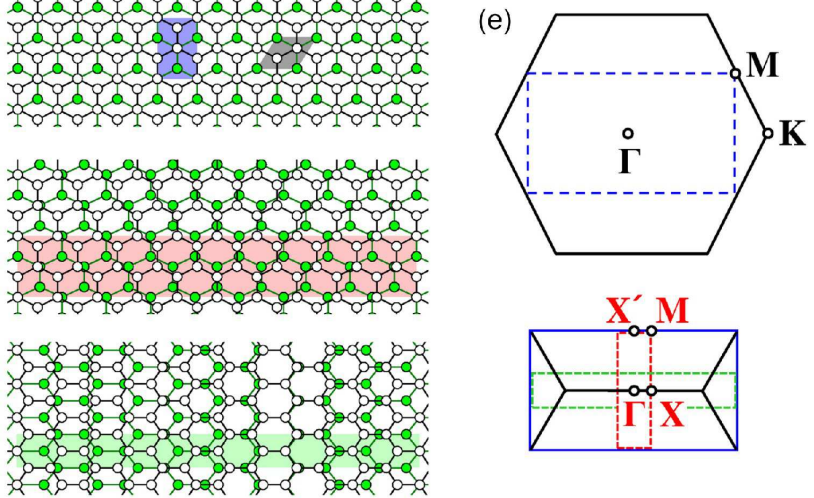

FIG. 1: (Color online) (a) Bilayer and single layer graphene junction between the source and drain. Red arrows: tensile force direction. (b) Schematic atomic structure of bilayer graphene at equilibrium, showing carbon atoms in the top layer (clear circles) and the bottom layer (green circles), and the bilayer graphene unit cells with four carbon atoms (grey parallelogram) and eight carbon atoms (blue rectangle). (c) Atomic structure of asymmetrically strained bilayer along the zigzag chain direction (AZS) with $11.1 \%$ strain on the bottom layer. (d) Asymmetrically strained bilayer in armchair direction (AAS) with $20 \%$ strain on the bottom layer. (e) Top panel: the first Brillouin zones (BZ) of bilayer graphene in equilibrium for the unit cell with four carbon atoms (black hexagon) and for the unit cell with eight carbon atoms (blue dotted rectangle). Bottom panel: first BZ for the AZS in (c) (red dotted rectangle); first BZ for AAS bilayer in (d) (green dotted rectangle).

effect is negligible when obtaining the energy bands using the present calculation scheme [26]. We note that present methods correctly describe the trend of the energy gap in bilayer graphene under external electric fields with a typical underestimate of the gap size compared with a recent optical measurement [20].

Homogeneous strains can be applied to the two layers of bilayer graphene asymmetrically in various device configurations. The prototypical system considered here is a junction between single-layer graphene and bilayer graphene [Fig. 1(a)]; this junction can be synthesized using the graphene transfer technique [27], and many mechanically exfoliated samples contain such junction geometries naturally [28]. If a uniform tensile force is applied to the junction [Fig. 1(a)], each layer in bilayer graphene experiences a different magnitude of homogeneous strain, because only one end of the top layer in bilayer graphene is pinched by the metallic lead whereas the other end is free-standing [Fig. 1(a)]. In this case, the top layer of bilayer graphene in the junction maintains its equilibrium atomic structure due to the negligible interlayer interaction along the in-plane direction [29], but the bottom layer is subjected to biaxial strain because both of its ends are clamped to the stretched substrates by the metallic leads [Fig. 1(a)]. Generally speaking, any device structure with asymmetric contacts to the top and bottom layers can produce asymmetric strains in each layer due to external tensile forces. Hereafter, we apply the periodic boundary condition to the asymmetrically strained bilayer graphene only. To satisfy the commensurability requirement for between the top and bottom layers along the direction of applied tensile force, the unit cell of the system must be a rectangle with a very high aspect ratio [Fig. 1(c) and 1(d)]. Accordingly, the first BZ of the strained graphene is folded into a small rectangle within the first BZ of the original bilayer graphene in equilibrium [Fig. 1(e)].

Here we considered two atomic configurations for asymmetrically strained bilayer graphene. Because pristine bilayer graphene has Bernal stacking between top and bottom layers [Fig. 1(b)], we apply strains along the two highly symmetric (i.e., zigzag and armchair) crystallographic directions of the hexagonal lattice of the bottom layer. First we fix the stacking order along the short edge of the unit cell, and then stretch the bottom layer along the normal to the edge. When the bottom layer is under strain along the zigzag-chain direction, we call this configuration the asymmetrically Z-strained bilayer graphene (AZS bilayer) [Fig. 1(c)]. When strain is applied along the armchair-chain direction in the bottom layer, we call it the asymmetrically A-strained bilayer graphene (AAS bilayer) [Fig. 1(d)]. We note that, in single-layer graphene, strains along those two directions induce different variations in its electronic structures and the effect of strain along arbitrary directions can be represented as a linear combination of them [8, 23].

In AZS bilayer graphene, an energy gap opens immediately when the bottom layer is stretched along the zigzag chain direction; the position where the energy gap opens in the first BZ shifts depends on the magnitude of strain and is not located at the highly symmetric points of the first BZ [Fig. 2]. The fundamental energy gap is indirect at strains up to $9 \%$, but direct at greater strains. In AAS bilayer graphene, no energy gap opening occurs up to a strain of about 14\% (not shown here). This shows that electronic band gap of asymmetrically strained bilayer graphene strongly depends on the direction of strain.

The energy gap of AZS bilayer graphene is created by strain-induced perpendicular electric fields that break the symmetry in the onsite energy of the top and bottom layers [11, 12]. The transverse electric fields across the two layers without any external electric source are possible because the increase of the work function of the strained bottom graphene generates a net charge transfer from the top layer to the bottom layer [Fig. 3]. The 


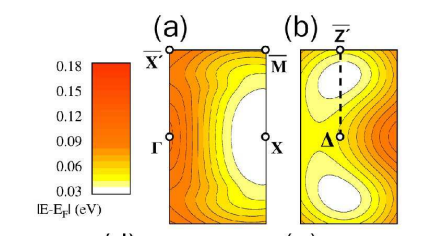

(d)

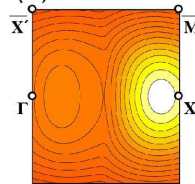

(g)

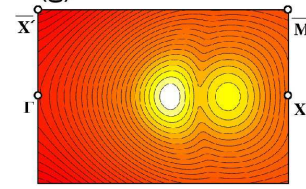

(h)

(e) (c)

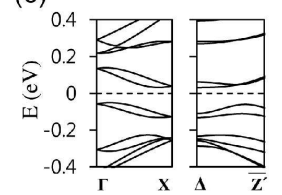

(f)
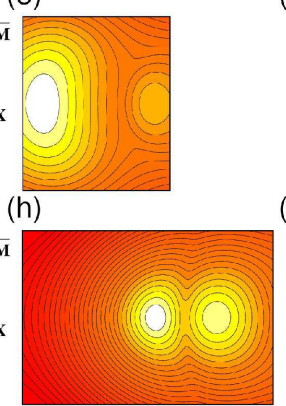

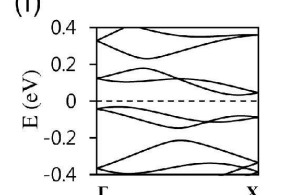

(i)

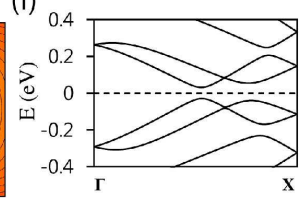

FIG. 2: (Color online) The energy contours for (a) the conduction band and (b) the valence band of the AZS bilayer with $4 \%$ strain on the bottom layer. Only contours for a part of the first BZ are drawn, where energy bands exhibit minimal and maximal values. Here, $\Gamma \bar{X}^{\prime}$ is one tenth of $\Gamma \mathrm{X}^{\prime}$ in the first BZ shown in Fig. 1(f). The scale bar for the contour is given with respect to the Fermi energy $\left(E_{F}\right)$ in units of eV. (c) the electronic band structure of the AZS bilayer with $4 \%$ strain (left panel) along the high symmetric line ( $\Gamma \mathrm{X})$ and (right panel) along the dotted line in (b) that crosses the k-points at which the minimum indirect band gap occurs. (d), (e) and (f) Energy contours and band structures for the AZS bilayer with $6.25 \%$ strain using the same convention as in (a), (b) and (c). (g), (h) and (i) Energy contours and bands for the AZS bilayer with $11.1 \%$ strain with the same previous convention.

electric fields shift and hybridize the conduction and valence bands, thereby opening energy gaps at the points at which band mixing occurs at the Fermi level. The gap opening in this system is thus a truly physical manifestation of the pseudo-scalar potential induced by mechanical deformations of graphene. In our calculations, the work function of single layer graphene increases almost linearly (0.4 eV by $14 \%$ strain) and accordingly, so does the net charge transfer between the two layers [Fig. 3(c)]. Due to the screening of the transferred charge, the net difference between local potentials in the two layers in bilayer graphene increases by $0.2 \mathrm{eV}$ for when asymmetric strain is $14 \%$ [Fig. 3(c)]. To see the change of onsite energies of each layer clearly, we calculate the effective potentials for electrons in the systems by substracting the total potential of each single-layer graphene from that of bilayer graphene and then taking average in the plane [right panels in Figs. 3 (a) and (b)]. The induced dipole field across the two layers is clearly visible when the lower layer is under the strain of $14.3 \%$ as shown in the right panel of Fig. 3 (b).

The indirectness of the energy gap in the AZS bilayer originates from the inequivalent formation of straininduced vector potentials in the two graphene layers. (a)
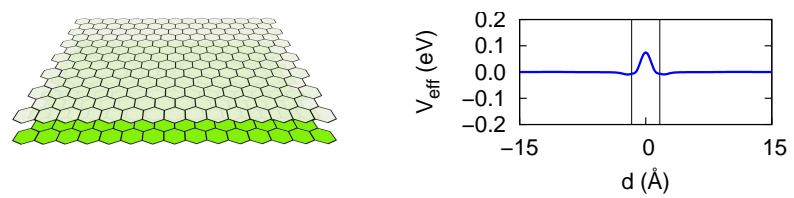

(b)
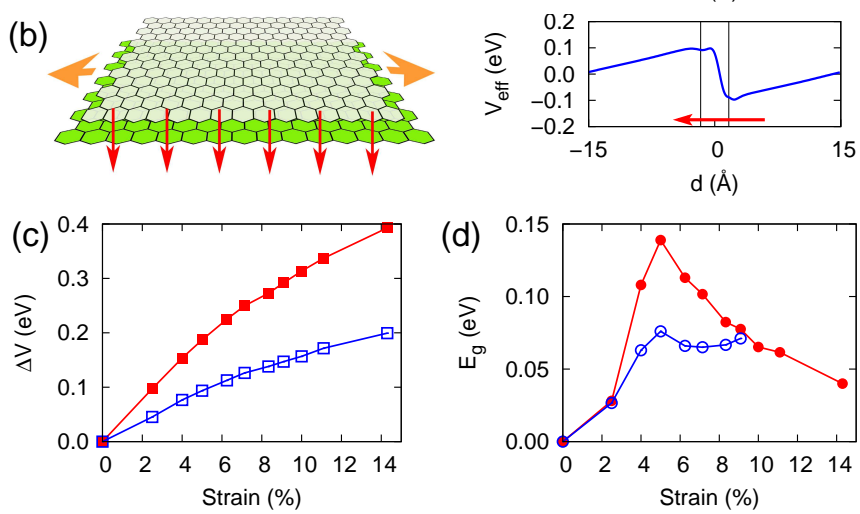

FIG. 3: (Color online) (a) Bilayer graphene in equilibrium (left panel) and the effective potential for electrons (right panel). Two vertical lines denote the position of bottom and top graphene layers, respectively. (b) Asymmetrically strained bilayer graphene with the bottom layer uniformly stretched along the orange arrows. A uniform electric field across the two layers (red arrows) is generated due to work function difference (left panel). The effective potential for electrons for AZS bilayer with $14.3 \%$ strain on the bottom layer (right) and corresponding electric field (red arrow) across the two layers are shown. (c) The work function difference (red) and the net potential difference (blue) between the top and bottom layers as a function of strain (\%) for the AZS bilayer. (d) The direct (red) and indirect (blue) energy gap of the AZS bilayer as a function of strain.

In single layer graphene, homogeneous strains shift the Dirac cones away from the high symmetric points in the first BZ, and this effect is equivalent to introducing a constant vector potential in the Dirac Hamiltonian [5, 8, 23]. The constant vector potential usually has no effect because it can be simply gauged away. In the present case, only the bottom layer has such a constant vector potential and it cannot be trivial. The displacement of Dirac cones in the strained bottom layer alters the points of band crossing between the conduction and valence bands of the bilayer, which should meet at the K-points of the first BZ of bilayer graphene without such asymmetric strains [5, 11]. Our calculations, suggest that asymmetrically strained bilayer graphene is unique in that both pseudo-scalar and pseudo-vector potentials induced by the strain open the energy gap and determine its characteristics.

The competition between various channels of interlayer interactions is yet another important factor that determines the size of the energy gap. All AAS bilayers and the highly strained AZS bilayer contain large domains of carbon hexagons that have almost a perfect overlap (AA stacking [30]) between pairs of layers [Fig. 1(d)]. In 
bilayer graphene with AA stacking, the low energy electrons behave as those in two interpenetrating Dirac cones of single layer graphene and no energy gap forms even with strong transverse external electric fields 31]. Likewise, when the interlayer interactions in the AA stacking dominate over all other interactions, the significant differences in the work function between the top and bottom layers are less effective in opening energy gaps than the cases with the Bernal stacking. For this reason, when the strain exceeds $9 \%$ in the AZS bilayer, the energy gap starts to decrease even though the net potential difference continues to increase. Moreover, when strain is applied along the armchair direction in the bottom layer of the AAS bilayer, the interlayer interaction in the AA stacking domain is predominant over all other channels and the energy gap does not open at all [Fig. 1(d)].

We find that as the strain in the bottom layer increases, the energy gap of the AZS bilayer first increases linearly, then decreases with a transition from an indirect gap to a direct gap. At a strain of $5 \%$, both the indirect energy gap $(0.076 \mathrm{eV})$ and the direct energy gap $(0.14 \mathrm{eV})$ are maximal [Fig. 3(d)]. This variation of energy gaps depending on the magnitude of asymmetric strains in bilayer graphene is quite in contrast to that observed in simple Bernal-type stacked bilayer graphene under external electric fields [11, 12, 14, 20]. The latter shows a monotonic increase and then saturation at an energy gap of $\sim 0.25 \mathrm{eV}$ due to screening of induced charges [11, 14, 20] as external fields increase. The unique interplay between atomic stacking and straininduced pseudo-scalar potential leads to the maximal energy gap at a certain magnitude and direction of strain. Hence, even under very gentle strains, the rectifying current due to the strain-induced energy gap should be measured in the single-layer/bilayer graphene junctions considered here. The atomic orientation of bilayer graphene and the size of applied strains could also be inferred from such measurements.

In summary, we show that one can control the energy gap of bilayer graphene only by the strength and direction of homogeneous strain within the experimentally accessible range [27, 32]. We show that, if homogeneous strains with different strengths are applied to each layer of bilayer graphene, transverse electric fields across the two layers can be generated without any external electronic sources, thereby opening an energy gap and enabling the system to behave as an electromechanical switch with tunable energy band gaps. This finding is a materialization of pseudo-vector and pseudo-scalar potentials induced by external mechanical perturbations in the relativistic massless Dirac Hamiltonians.

We thank H. J. Lee, B. H. Hong, and H.-Y. Kee for discussions and comments and specially thank S. Y. Jung for discussions on device geometries. S.-M. C. and S.-H. J. were supported by the National Research Foundation of Korea (NRF) grant funded by the Korea government
(MEST) (Grant No. 2009-0087731 and WCU program No. R31-2008-000-10059-0). Y.-W. S. was supported by the NRF grant funded by MEST (Quantum Metamaterials research center, No. R11-2008-053-01002-0 and Nano R\&D program 2008-03670).

* Electronic address: jhish@postech.ac.kr

$\dagger$ Electronic address: hand@kias.re.kr

[1] V. H. Crespi, M. L. Cohen, and A. Rubio, Phys. Rev. Lett. 79, 2093 (1997).

[2] T. W. Tombler et. al., Nature 405, 769 (2000).

[3] K. S. Novoselov et. al., Nature 438, 197 (2005).

[4] Y. Zhang, Y.-W. Tan, H. L. Stormer and P. Kim, Nature 438, 201 (2005).

[5] A. H. Castro Neto, F. Guinea, N. M. R. Peres, K. S. Novoselov and A. K. Geim, Rev. Mod. Phys. 81, 109 (2009).

[6] M. M. Fogler, F. Guinea and M. I. Katsnelson, Phys. Rev. Lett. 101, 226804 (2008).

[7] V. M. Pereira, V. M. and A. H. Castro Neto, Phys. Rev. Lett. 103, 046801 (2009).

[8] V. M. Pereira, A. H. Castro Neto and N. M. R. Peres, Phys. Rev. B 80, 045401 (2009).

[9] F. Guinea, M. I. Katsnelson and A. K. Geim, Nature Phys. 6, 30 (2009).

[10] F. Guinea, A. K. Geim, M. I. Katsnelson and K. S. Novoselov, Phys. Rev. B 81, 035408 (2010).

[11] E. McCann, Phys. Rev. B 74, 161403(R) (2006).

[12] T. Ohta et. al., Science 313, 951 (2006).

[13] Y.-W. Son, M. L. Cohen and S. G. Louie, Nature 444, 347 (2006).

[14] H. Min, B. Sahu, S. K. Banerjee and A. H. MacDonald, Phys. Rev. B 75, 155115 (2007).

[15] E. V. Castro et. al., Phys. Rev. Lett. 99, 216802 (2007).

[16] J. B. Oostinga et. al., Nature Mat. 7, 151 (2008).

[17] A. B. Kuzmenko et. al., Phys. Rev. Lett. 103, 116804 (2009).

[18] K. F. Maik, C. H. Lui, J. Shan, and T. F. Heinz, Phys. Rev. Lett. 102, 256405 (2009).

[19] Z. Q. Li et. al., Phys. Rev. Lett. 102, 037403 (2009).

[20] Y. Zhang et. al., Nature 459, 820 (2009).

[21] S. Ono and K. Sugihara, J. Phys. Soc. Jpn. 21, 861 (1966).

[22] H. Suzuura and T. Ando, Phys. Rev. B 65, 235412 (2002).

[23] S.-M. Choi, S.-H. Jhi and Y.-W. Son, Phys. Rev. B, 81, 081407(R) (2010).

[24] G. Kresse and J. Hafner, Phys. Rev. B 49, 14251 (1994).

[25] D. M. Ceperley and B. J. Alder, Phys. Rev. Lett. 45, 566 (1980).

[26] V. Barone et. al., J. Comput. Chem. 30, 934 (2008).

[27] K. S. Kim et. al., Nature 457, 706 (2009).

[28] P. Blake et. al., Appl. Phys. Lett. 91, 063124 (2007).

[29] A. N. Kolmogorov and V. H. Crespi, Phys. Rev. Lett. 85, 065503 (2004).

[30] S. Horiuchi et. al., Jpn. J. Appl. Phys. 42, L1073 (2003).

[31] B. R. K. Nanda and S. Satpathy, Phys. Rev. B 80, 165430 (2009).

[32] S. Bae et. al. Nat. Nanotechnol. 5, 574 (2010). 